\title{
Nitric Oxide Metabolite Concentration in Cerebrospinal Fluid: Useful as a Prognostic Marker?
}

\author{
Saurabh Singh ${ }^{1}$, Jyoti Prakash ${ }^{1}$, Rakesh Singh $^{2}$, Ashish Verma $^{3}$, Hemant Bansal $^{1}$ \\ ${ }^{1}$ Department of Orthopaedics, Institute of Medical Sciences, Banaras Hindu University, Varanasi, India \\ ${ }^{2}$ Department of Biochemistry, Institute of Medical Sciences, Banaras Hindu University, Varanasi, India \\ ${ }^{3}$ Department of Radiology, Institute of Medical Sciences, Banaras Hindu University, Varanasi, India
}

\begin{abstract}
Study Design: Prospective study.
Purpose: To establish the significance of cerebrospinal fluid (CSF) nitric oxide metabolite (NOx) concentration in acute spinal cord injury (SCI) patients to assess the neurological severity and prognosis.

Overview of Literature: Quantitative analysis of specific biomarkers in CSF will assess neurological severity more accurately and permit the formulation of a more precise management plan.

Methods: Forty SCI patients represented the cases and 20 lower limb injury patients were the controls. NOx concentration in CSF was measured at week 1, 2, and 4 by Griess method. Magnetic resonance imaging (MRI, T2-weighted) done in each case to measure cord edema and neurological severity was assessed using the Frankel classification.

Results: CSF NOx concentration peaked at week 2 and declined to normal by week 4 . The concentration remained normal in controls. Mean NOx concentration was directly proportional to the severity of acute $\mathrm{SCl}$ as correlated with cord edema seen in MRI and neurological severity assessed.

Conclusions: CSF NOx concentration can be considered a specific quantitative biomarker in acute stage of SCI to predict the severity and prognosis of SCl patients.
\end{abstract}

Keywords: Nitric oxide; Cord edema; Cerebrospinal fluid; Magnetic resonance imaging; Neurological severity; Recovery

\section{Introduction}

Morbidity and mortality is a major concern in spinal cord injury (SCI). It usually has a phase of spinal shock precipitated immediately after the primary mechanical injury followed by a secondary injury. The secondary injury is due to infiltration of leukocytes, oxidative stress, tissue edema and neuronal ischemia. The assessment of injury severity can be done clinically by Frankel grading.

Radiologically, magnetic resonance imaging (MRI) findings in spinal cord after injury can also predict the prognosis, but a specific quantitative biomarker can help in assessing the neurological severity and prognosis more precisely and aids in planning appropriate management.

Secondary injury causes apoptosis of neurons and nerve degeneration [1,2]. Nitric oxide metabolite (NOx) works in secondary injury. It is involved in neuronal signal transmission and causes oxidative stress related cytotoxic effects and neuron apoptosis secondary to nerve degeneration and dysfunction $[2,3]$. Its relation with SCI severity

Received Dec 24, 2015; Revised Feb 3, 2016; Accepted Feb 24, 2016

Corresponding author: Jyoti Prakash

Department of Orthopaedics Surgery, Institute of Medical Sciences, Banaras Hindu University, Varanasi, 221005 U.P, India

Tel: +91-78-3916-6976, Fax: +91-33-2355-7214, E-mail: prakash_jyoti31@yahoo.com 
has not been explored much in humans. We have examined the cerebrospinal fluid (CSF) concentration of NOx and correlated it with the clinical and MRI findings The prognosis of SCI is usually predicted based on the early results of MRI [4]; i.e., intramedullary signal alterations due to hematoma, or edema in the spinal cord, as well as the Frankel et al. classification [5,6]. Early quantitative prediction of the prognosis is not easy, however, because initial paresis due to spinal cord shock can change with time.

There are currently no quantitative predictors for neurologic improvement at the acute stage. So we have examined the CSF concentration of NOx and compared it with the clinical and MRI findings to assess the neurological severity, prognosis, and planning of surgical timing and clarifying prediction of recovery.

\section{Materials and Methods}

\section{Case}

Forty patients (mean age, 38 years) with SCI at different levels were taken after obtaining written informed consent. The cases were followed up for a minimum of 12 months. All the cases had complaint of motor weakness, numbness and sensory loss with or without bladder and bowel involvement initially after injury. The mode of injury included 34 fall from height, 12 road traffic accident and 4 railway accident patients treated at Sir Sunderlal Hospital, Banaras Hindu University. Patients with history of associated head injury were not included in the study.

\section{Control}

The control group consisted of 20 admitted patients with lower limb injury having different types of closed fracture (10 male and female patients, each ranging from 18 to 50 years of age; mean age, 35 years).

\section{Collection of CSF}

CSF ( $1 \mathrm{~mL})$ was collected using a $25 \mathrm{G}$ needle under aseptic conditions in all the cases within 30 days of injury. Twenty cases of Frankle (A and B) and 20 cases of Frankel (C and D) were included. In 15 cases, CSF was taken three times at 0-7 days, 10-14 days and 20-28 days to measure change in concentration of NOx with time. CSF was collected in the control group was taken within 30 days of injury at the time of spinal anesthesia before surgery. The specimens were stored at $-20^{\circ} \mathrm{C}$ for measurement of NOx.

\section{Measurement of NOX}

NOx was measured with the Griess method. The minimum detectable NO concentration was $0.05 \mu \mathrm{m} / \mathrm{L}$. The CSF sample was first deproteinized and centrifuged then the assay was performed using a NO analyser that was calibrated using a standard solution prior to separation of $\mathrm{NO} 2$ and NO3. NO3 was reduced to NO2 to which Griess reagent was added. It formed a purple azodye. The absorption was measured at $540 \mathrm{~nm}$ using a spectrometer. The concentration was measured at the peak area of absorption. The accuracy of this method is well documented.

\section{Measurement of cord edema}

MRI was done in all the cases. The T2 sagittal section weighted sequences were studied for measurement of cord edema length within the spinal cord [7]. The extent of edema in both the ends of the cord was marked and the results were measured in MRI consoles. This showed the number of segments the cord edema had spread. This approach has the highest correlation with patient prognosis as compared to axial sequences [7].

\section{Evaluation of neurological severity and recovery}

Assessment of severity was done using Frankel Classification A, B, C, D, and E (based on sensory and motor involvement) at the time of reporting and after final followup. Frankel A and B were included in one group and C and $\mathrm{D}$ were included in the other group. Assessment of recovery was done in all 40 cases by Frankel classification at the time of final follow-up. The recovery was calculated and compared.

\section{Data analyses}

All the data are in mean \pm standard deviation. Correlation was explored by Spearmans ranked correlation coefficient. A $p<0.05$ was taken as statistically significant. The difference between the two groups was tested using the 
Mann-Whitney $U$ test. The NOx concentration differences among the various time periods (first week, second week, and fourth week) consecutively in 15 cases were tested using the Wilcoxon rank sum test.

Table 1. Difference in NOx level in 15 cases where CSF examination done in serial interval thrice

\begin{tabular}{|c|c|c|c|}
\hline \multirow{2}{*}{$\begin{array}{l}\text { Serial } \\
\text { no. }\end{array}$} & \multicolumn{3}{|c|}{ Nitric oxide level $(\mu \mathrm{m})$} \\
\hline & 1st week & 2nd week & 4th week \\
\hline 1. & 9.2 & 92 & 9 \\
\hline 2. & 9.6 & 69 & 8.6 \\
\hline 3. & 8.7 & 61 & 10.4 \\
\hline 4. & 6 & 88 & 8.5 \\
\hline 5. & 9.4 & 101 & 7.8 \\
\hline 6. & 8.5 & 92 & 9.2 \\
\hline 7. & 7.5 & 61 & 7.6 \\
\hline 8. & 6.59 & 72 & 10 \\
\hline 9. & 8 & 73 & 8 \\
\hline 10. & 9.2 & 81 & 8.6 \\
\hline 11. & 8.7 & 72.5 & 9 \\
\hline 12. & 7.1 & 75.5 & 8.2 \\
\hline 13. & 7.5 & 70 & 8 \\
\hline 14. & 8.7 & 85 & 8.4 \\
\hline 15. & 7.4 & 79 & 7.6 \\
\hline Mean \pm SD & $8.31 \pm 0.91$ & $78.13 \pm 11.65$ & $8.59 \pm 0.82$ \\
\hline
\end{tabular}

NOx, nitric oxide metabolite; CSF, cerebrospinal fluid; SD, standard deviation.

\section{Results}

The mean NOx concentration in week 1,2, and 4, and in the control group was $8.31 \pm 0.91,78.13 \pm 11.65,8.59 \pm 0.82$ and $4.52 \pm 0.79 \mu \mathrm{m}$, respectively. Differences in NOx at week 1, 2 and 4 in the 15 consecutive cases (Table 1, Fig. 1) were tested using the Wilcoxon rank sum test with $r=0.868$ and $p \geq 0.001$. The patients with a higher concentration of NOx in CSF of group Frankel $(A+B ; n=20)$ had a mean of $84 \pm 14.09 \mu \mathrm{m}$; the average number of segments involved was three. Frankel $(\mathrm{C}+\mathrm{D} ; \mathrm{n}=20)$ patients displayed a mean NOx (Tables 2, 3) concentration of $9.68 \pm 2.00 \mu \mathrm{m}$ with an average of 1.2 involved segments (Tables 4,5 ).

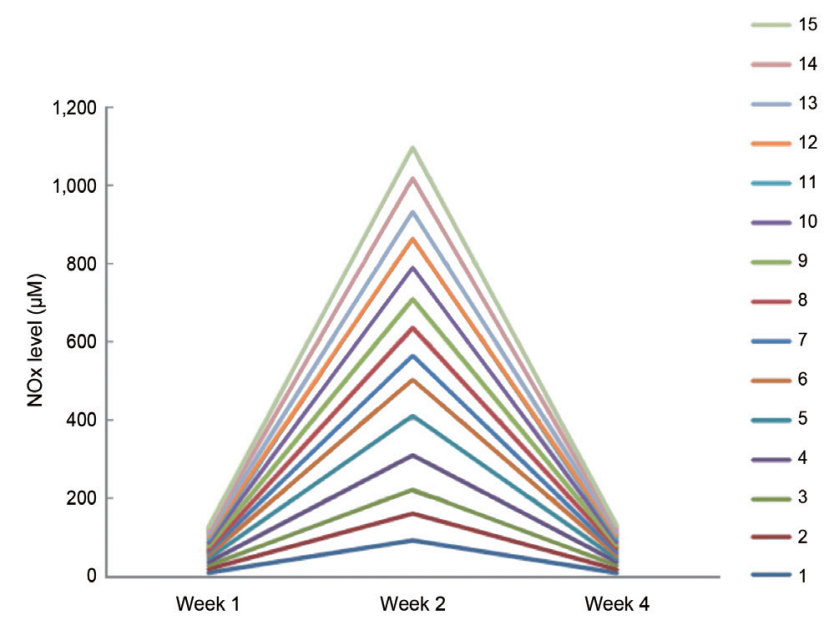

Fig. 1. Chart depicting time-course of changes in NOx concentration at week 1, 2, and 4 after $\mathrm{SCl}$ in 15 consecutive cases. NOx, nitric oxide metabolite; $\mathrm{SCl}$, spinal cord injury.

Table 2. NOx concentration in Frankel grade $A+B$ and $C+D$

Frankel grade NOx level $(\mu \mathrm{m}$, mean \pm SD) $\quad$ Mann-Whitney $U$ test ( $p$-value)

$\begin{array}{ccc}A+B & 84.00 \pm 14.09 & <0.001 \\ C+D & 9.68 \pm 2.00 & <0.001\end{array}$

NOx, nitric oxide metabolite; SD, standard deviation.

Table 3. Recovery percentage in Frankel grade $A+B$ and $C+D$

\begin{tabular}{|c|c|c|c|c|c|c|c|}
\hline \multirow[b]{2}{*}{ Initial exam } & \multirow{2}{*}{$\begin{array}{l}\text { Total } \\
\text { cases }\end{array}$} & \multirow{2}{*}{$\begin{array}{l}\text { Mean NOx level } \\
\text { in week } 2(\mu \mathrm{m})\end{array}$} & \multicolumn{4}{|c|}{ Final follow-up } & \multirow{2}{*}{$\begin{array}{c}\text { Recovery } \\
(\%)\end{array}$} \\
\hline & & & $(A+B)$ & $(C+D)$ & E & $\begin{array}{c}\text { Not } \\
\text { reviewed }\end{array}$ & \\
\hline Frankel (A+B) & 20 & $84.00 \pm 14.09$ & 13 & 4 & 2 & 1 & 10 \\
\hline Frankel (C+D) & 20 & $9.68 \pm 2.00$ & 0 & 7 & 10 & 3 & 50 \\
\hline
\end{tabular}

NOx, nitric oxide metabolite. 
Table 4. FrankeL (A+B) group showing NOx level in CSF and MRI findings

\begin{tabular}{|c|c|c|}
\hline Serial no. & $\begin{array}{l}\text { Nitric oxide level } \\
\text { in } \operatorname{CSF}(\mu \mathrm{m})\end{array}$ & $\begin{array}{l}\text { Involved segments } \\
\text { (cord edema) }\end{array}$ \\
\hline 1. & 110 & 3 \\
\hline 2. & 88 & 3 \\
\hline 3. & 104 & 3 \\
\hline 4. & 85 & 2 \\
\hline 5. & 105 & 3 \\
\hline 6. & 92 & 3 \\
\hline 7. & 73 & 3 \\
\hline 8. & 72.5 & 3 \\
\hline 9. & 75.5 & 3 \\
\hline 10. & 69 & 2 \\
\hline 11. & 61 & 3 \\
\hline 12. & 79 & 3 \\
\hline 13. & 101 & 3 \\
\hline 14. & 81 & 2 \\
\hline 15. & 96 & 3 \\
\hline 16. & 72 & 3 \\
\hline 17. & 70 & 3 \\
\hline 18. & 69 & 3 \\
\hline 19. & 85 & 3 \\
\hline 20. & 92 & 4 \\
\hline Mean \pm SD & $84.00 \pm 14.09$ & 3 \\
\hline
\end{tabular}

NOx, nitric oxide metabolite; CSF, cerebrospinal fluid; MRI, magnetic resonance imaging; $S D$, standard deviation.

\section{Discussion}

\section{Time-course of changes in NOx concentration after SCI}

Consistent with findings from cauda equina injury, several reports indicated that inducible nitric oxide synthase (iNOS) expression and NO concentration significantly increase around the injured spinal cord [8]. Hamada et al. [9] demonstrated that iNOS mRNA at the SCI lesion peaks 24 hours after SCI. Xu et al. [10] reported a progressive increase in iNOS expression in the injured spinal cord with maximal expression on day 7, with high levels of iNOS expression persisting for 2 weeks after SCI $[5,6]$. With regard to production of NOx, Nakahara et al. [8] used the same system that we used in the present study, and reported that NOx increases with biphasic changes, i.e in between 1-12 hours and between 24 hours-3 days. Fujieda et al. [11] demonstrated that $\mathrm{N}$-acetyl aspartate
Table 5. Frankel $(\mathrm{C}+\mathrm{D})$ group showing NOx level in CSF and MRI findings

$\begin{array}{ccc}\text { Serial no. } & \begin{array}{c}\text { Nitric oxide level } \\ \text { in CSF }(\mu \mathrm{m})\end{array} & \begin{array}{c}\text { Involved segments } \\ \text { (cord edema) }\end{array}\end{array}$

\begin{tabular}{|c|c|c|}
\hline 1. & 8.5 & 1 \\
\hline 2. & 12.2 & 1 \\
\hline 3. & 10.5 & 1 \\
\hline 4. & 8 & 1 \\
\hline 5. & 9.2 & 1 \\
\hline 6. & 10.6 & 2 \\
\hline 7. & 10 & 1 \\
\hline 8. & 11 & 2 \\
\hline 9. & 13 & 2 \\
\hline 10. & 11 & 2 \\
\hline 11. & 7.5 & 1 \\
\hline 12. & 7.8 & 1 \\
\hline 13. & 8 & 1 \\
\hline 14. & 7.7 & 1 \\
\hline 15. & 8 & 1 \\
\hline 16. & 14 & 1 \\
\hline 17. & 8.2 & 1 \\
\hline 18. & 7.5 & 1 \\
\hline 19. & 12 & 1 \\
\hline 20. & 9 & 1 \\
\hline Mean $\pm S D$ & $9.68 \pm 2.00$ & 1.2 \\
\hline
\end{tabular}

NOx, nitric oxide metabolite; CSF, cerebrospinal fluid; MRI, magnetic resonance imaging; SD, standard deviation.

(NAA) level in the spinal cord might provide a meaningful biomarker in rats. These animal studies support the finding that CSF NOx increases from 1 to 14 days after SCI. Several animal experiments have established the role of NO in SCI. In rats, iNOS level correlates significantly with SCI, peaking at 24 hours after the injury and declining to normal after 72 hours. Xu et al. [10] reported that NO concentration reaches a maximum on day 7 after SCI, persists until 2 weeks, then declines. NO 0 level features a biphasic peak in the first 12 hours and between days 1-3.

In our study, the NOx concentration increased minimally to a mean $(8.31 \pm 0.91 \mu \mathrm{m})$ in week 1 (acute phase), increases significantly to a mean of $78.13 \pm 11.65 \mu \mathrm{m}$ in week 2 (sub-acute phase) during tissue phagocytosis and returned to near normal (mean $8.59 \pm 0.82 \mu \mathrm{m}$ in week 4 . Differences in NOx at week 1, 2, and 4 in 15 consecutive cases were tested using the Wilcoxon rank sum test. NOx was cytotoxic in the tissue and iNOx displayed specific 
cytotoxic effects in the spinal cord after injury. The oxidized form is reportedly more toxic to proteins, lipids and DNA, and induces neuronal apoptosis, which causes neuronal degeneration and dysfunction in the spinal cord. This suggests that in the sub-acute phase the increase in NOx concentration level in CSF correlates with disease activity according to the severity calculated by the Frankel classification and American Spine Injury Association Score motor score. Presently, the concentration of NOx was high in cases with more severe injury. The concentration of NOx was time-dependent, with a peak value in week 2 of SCI.

The collective findings indicate that the NOx acts as a specific indicator and marker of severity of neurological impairment in SCI.

\section{NOx concentration indicates the severity of neurologic impairment}

The NOx in intraventricular CSF, also increases in patients with severe closed head injury, peaking at 30 to 42 hours after trauma, and is greater in patients that die compared to surviving patients. This finding is roughly consistent with our data in SCI; in our study NOx in Frankel $(\mathrm{A}+\mathrm{B})$ groups were greater than that in Frankel $(\mathrm{C}+\mathrm{D})$ groups, except that the peak NOx occurred during the early subacute stage (week 2). In multiple sclerosis, moreover, CSF nitrite levels correlate with clinical disease activity, suggesting that nitrite levels might be a useful surrogate marker for disease activity in multiple sclerosis. In the present study, there was a significant correlation between the NOx at the early sub acute stage and Frankel severity scoring. These findings suggest that NOx at the early subacute stage is an indicator of the severity of neurologic impairment in SCI.

\section{Correlation between CSF NOx concentration and MRI cord edema}

The CSF NOx concentration taken at week 2 in 20 cases 116 of Frankel group $(\mathrm{A}+\mathrm{B})$ and 20 cases of Frankel $(\mathrm{C}+\mathrm{D})$ was compared with the MRI cord edema (involved segments). It shows that the patients with more concentration of NOx in CSF of group Frankle $(A+B)$ had a mean of $84 \pm 14.09 \mu \mathrm{m}$ with an average of 3 involved (Table 4 ), and the group Frankel $(\mathrm{C}+\mathrm{D})$ had a mean NOx concentration of $9.68 \pm 2.00 \mu \mathrm{m}$ with an average of one involved segment
(Table 5). The difference in means between the two groups was tested using the Mann-Whitney U test. Both the groups were compared and correlations were determined by Spearman ranked correlation coefficient. The $p$-value of $\leq 0.05$ was considered statistically significant. This study and comparison shows a significant positive correlation with a correlation coefficient of $p<0.001$ between the MRI cord edema and CSF NOx in sub acute phase with $r=0.868$. MRI cord edema and cord hemorrhage has already proven to be a good prognostic diagnostic tool in SCI. So, CSF NOx can be a quantitative prognostic biomarker in SCI.

\section{Significant correlation between CSF NOx concentration and neurologic recovery in SCI}

$\mathrm{NO}$ reacts with $\mathrm{O} 2$ to form the powerful oxidant peroxynitrite (ONOO-) [12], which directly oxidizes lipids, DNA and proteins. Excessive NO produced by iNOS has cytotoxic effects and induces neuronal apoptosis in vitro, in vivo and in the white matter around the SCI site in humans, causing neural degeneration and neuro-dysfunction in the spinal cord. These data suggest that the high NOx concentration may result in poor treatment results in some neurologic diseases. Preoperative CSF NOx concentration is higher than in pain-free controls and is significantly correlated with the degree of pain relief following surgery for lumbar degenerative disease and with the neurologic recovery rate after decompression surgery for cervical degenerative disease [13]. Frankel's classification at the acute stage is a predictor of cases with Frankel A, 31.3\% of cases with Frankel B and $70.7 \%$ of cases with Frankel C recovered to at least Frankel D at the 1-year follow-up in 1461 SCI cases in an earlier study. In the present study, only $5 \%$ of cases recovered from Frankel $(A+B)$ to $E$ where as $40 \%$ cases recovered from Frankel $(\mathrm{C}+\mathrm{D})$ to E. CSF leukotriene $\mathrm{C} 4$, which is a metabolite of arachidonic acid (eicosanoids), levels increase at the acute stage and correlate with the severity of neurologic impairment in patients with SCI. To our knowledge, however, there are no quantitative predictors for neurologic recovery in SCI at the acute stage. The present study indicates that (NOx) at the early sub-acute stage (approximately week 2) after trauma significantly correlates with neurologic recovery in SCI.

In this study, after 12 months of follow-up out of 20 patients classified under Frankle $(\mathrm{A}+\mathrm{B})$ only 4 patients improved to Frankel (C+D) but 2 to Frankle (E), 13 cases 
still did not recovered from Frankel $(\mathrm{A}+\mathrm{B})$ with only $10 \%$ recovery and in 20 cases of FrankeL $(\mathrm{C}+\mathrm{D})$ group, 10 cases improved to FrankeL (E) with 7 cases still remaining as in Frankel $(\mathrm{C}+\mathrm{D})$ with $50 \%$ recovery. Also, in the present study we have found that cases with cord edema more than 3 segments involvement was in the Frankel $(A+B)$ group and have poor prognosis where as cases with cord edema less than 3 segments was in the Frankel $(C+D)$ group, and had a good prognosis.

In the present study, the NOx concentration correlated significantly with the neurological recovery in early subacute phase in week 2 . The NOx concentration was compared and correlated with the MRI findings (segments involved due to cord edema) to assess its specificity in SCI. NOx concentration at week 2 following SCI can be taken as a possible quantitative predictor for neurological recovery.

\section{Limitations of the study}

The most important limitation of this study is the imbalance in the severity of impairment of neurological status. The other drawback is the exact calculation of rostrocordal edema in the patients and accurate time to perform MRI within the acute period cannot be determined, so it is done as early as possible.

\section{Conclusions}

The severity of injury can be calculated by estimating the NOx level in CSF in the sub-acute phase. A significant positive correlation was evident between the MRI cord edema and CSF NOx in the sub-acute phase. CSF NOx is implicated as a good quantitative prognostic biomarker in SCI and can be a possible quantitative predictor for neurological recovery. In India where the socioeconomic status is low the NOx estimation in SCI patient will be a good option for assessment of severity and prognosis and for planning of surgery.

\section{Conflict of Interest}

No potential conflict of interest relevant to this article was reported.

\section{References}

1. Tator CH. Spine-spinal cord relationships in spinal cord trauma. Clin Neurosurg 1983;30:479-94.

2. Tator $\mathrm{CH}$. Update on the pathophysiology and pathology of acute spinal cord injury. Brain Pathol 1995; 5:407-13.

3. Keene JS, Zdeblick TA. Physiological consequences and assessment of injury to the spinal cord. In: Zejdlik CP, editor. Management of spinal cord injury. Boston: Jones and Bartlett Publishers; 1992. p.53-86.

4. Kulkarni MV, Bondurant FJ, Rose SL, Narayana PA. 1.5 tesla magnetic resonance imaging of acute spinal trauma. Radiographics 1988;8:1059-82.

5. Kimura S, Watanabe K, Yajiri Y, et al. Cerebrospinal fluid nitric oxide metabolites in painful diseases. Neuroreport 1999;10:275-9.

6. Kimura S, Watanabe K, Yajiri Y, et al. Cerebrospinal fluid nitric oxide metabolites are novel predictors of pain relief in degenerative lumbar diseases. Pain 2001;92:363-71.

7. Goldberg AL, Rothfus WE, Deeb ZL, et al. The impact of magnetic resonance on the diagnostic evaluation of acute cervicothoracic spinal trauma. Skeletal Radiol 1988;17:89-95.

8. Nakahara S, Yone K, Setoguchi T, et al. Changes in nitric oxide and expression of nitric oxide synthase in spinal cord after acute traumatic injury in rats. J Neurotrauma 2002;19:1467-74.

9. Hamada Y, Ikata T, Katoh S, et al. Roles of nitric oxide in compression injury of rat spinal cord. Free Radic Biol Med 1996;20:1-9.

10. Xu J, Kim GM, Chen S, et al. iNOS and nitrotyrosine expression after spinal cord injury. J Neurotrauma 2001;18:523-32.

11. Fujieda Y, Ueno S, Ogino R, et al. Metabolite profiles correlate closely with neurobehavioral function in experimental spinal cord injury in rats. PLoS One 2012;7:e43152.

12. Fehlings MG, Sekhon LH. Cellular, ionic and biomolecular mechanisms of the injury process. In: Tator $\mathrm{CH}$, Benzel EC, Committee AP, editors. Contemporary management of spinal cord injury: from impact to rehabilitation. Park Ridge: American Association of Neurological Surgeons; 2000. p.33-50.

13. Hosaka N, Kimura S, Yamazaki A, et al. Significant correlation between cerebrospinal fluid nitric oxide concentrations and neurologic prognosis in incomplete cervical cord injury. Eur Spine J 2008;17:281-6. 